\title{
The $q$-Mellin transform of automorphic forms and converse theorems
}

by

\author{
Mitsugu Mera (Fukuoka)
}

1. Introduction. It is well known in the classical Hecke theory that the completed L-functions defined by a Mellin transform of automorphic forms can be meromorphically continued to the whole complex plane $\mathbb{C}$, and satisfy a functional equation. Furthermore, we have the so-called converse theorems established by Hecke [5] and Weil [10] which characterize the image of the Mellin transform of automorphic forms. The purpose of this paper is to establish $q$-analogues of the theory by replacing the Mellin transform with the $q$-Mellin transform which is given by the Jackson integral (see, e.g., [2]).

Let $0<q<1$. We call $f:(0, \infty) \rightarrow \mathbb{C}$ a moderate asymptotic function of order $\left(i_{0}, j_{0}\right)$ if $f$ is continuous, $f(y)=O\left(y^{i_{0}}\right)$ as $y \rightarrow 0$ and $f(y)=O\left(y^{j_{0}}\right)$ as $y \rightarrow \infty$ for some $i_{0}, j_{0} \in \mathbb{R}\left(i_{0}>j_{0}\right)$. Then the $q$-Mellin transform (Jackson-Mellin's transform) of $f$ is defined by

$$
\begin{aligned}
M_{q}(f)(s) & :=\int_{0}^{\infty} y^{s-1} f(y) d_{q} y=\sum_{n \in \mathbb{Z}} q^{n(s-1)} f\left(q^{n}\right)\left(q^{n}-q^{n+1}\right) \\
& =(1-q) \sum_{n \in \mathbb{Z}} q^{n s} f\left(q^{n}\right)
\end{aligned}
$$

for $-i_{0}<\operatorname{Re}(s)<-j_{0}$. It becomes the classical Mellin transform $M(f)(s)$ $:=\int_{0}^{\infty} y^{s-1} f(y) d y$ when $q \rightarrow 1$.

According to [4], when $F$ is a full-modular form (i.e., a modular form for $\mathrm{SL}(2, \mathbb{Z}))$, the $q$-series $\Lambda_{q}(s, F)$ defined by the $q$-Mellin transform of $F$ has a meromorphic continuation to $\mathbb{C}$ and satisfies a functional equation. This is why it is a natural question whether a converse result can hold.

More generally, we describe properties of the $q$-Mellin transform of automorphic forms for congruence subgroups and determine their $q$-Mellin

2000 Mathematics Subject Classification: Primary 11F66.

Key words and phrases: Mellin's transform, Jackson's integral, automorphic forms, $q$-series. 
images. The classical converse theorem needs the Mellin inversion formula in its proof, while the proof of the $q$-analogue of the converse theorem needs an inversion formula for the $q$-Mellin transform. Note that the $q$-Mellin inversion formula does not involve $F(i y)$ for $y \neq q^{n}$. Thus, in our proofs of converse theorems, we need to assume analytic properties of $\Lambda_{q}(s, F)$ for infinitely many $q \in(0,1)$.

The paper is organized as follows. In Section 2, we show properties of the $q$-Mellin transform of automorphic forms for congruence groups. In Section 3, we show two converse theorems which are the main results of this paper. They correspond to the cases of full-modular forms and of automorphic forms for congruence groups. In Section 4, as an application, we recall the $q$-analogue of the complete Riemann zeta function $\zeta^{(q)}(s)$ introduced in [3] and [4], and give two characterizations of the image of the $q$-Mellin transform of the Jacobi theta function $\vartheta(z):=\sum_{m \in \mathbb{Z}} e^{\pi i m^{2} z}$, which is an automorphic form of weight $1 / 2$ with a suitable multiplier system. Note that $\zeta^{(q)}(s)$ is given by the $q$-Mellin transform of $\vartheta\left(i y^{2}\right)-1$. In Appendix A, we show another inversion formula. In Appendix B, we prove that $\zeta^{(q)}(s)$ actually gives a true $q$-analogue, that is, we show that the classical limit $q \rightarrow 1$ of $\zeta^{(q)}(s)$ produces the complete Riemann zeta function not only for $\operatorname{Re}(s)>1$ but for all $s \in \mathbb{C}$.

Throughout this paper, we denote the field of complex numbers, that of real numbers, the ring of rational integers and the set of positive integers by $\mathbb{C}, \mathbb{R}, \mathbb{Z}$ and $\mathbb{N}$ respectively. Also, we denote the upper half-plane $\{z \in \mathbb{C} \mid$ $\operatorname{Im}(z)>0\}$ by $\mathbb{H}$.

\section{The $q$-Mellin transform of automorphic forms for congruence} groups. Let $k, N \in \mathbb{N}$. Let

$$
\omega_{N}:=\left(\begin{array}{rr}
0 & -1 \\
N & 0
\end{array}\right)
$$

and $\chi$ be a Dirichlet character modulo $N$ with $\chi( \pm 1)=( \pm 1)^{k}$. Denote by $\mathcal{M}_{k}\left(\Gamma_{0}(N), \chi\right)$ the space of holomorphic automorphic forms of weight $k$ for the congruence group

$$
\Gamma_{0}(N):=\left\{\gamma \in \operatorname{SL}(2, \mathbb{Z}) \mid \gamma \equiv\left(\begin{array}{ll}
* & * \\
0 & *
\end{array}\right)(\bmod N)\right\}
$$

with a character $\chi$. Then we have the following:

Proposition 2.1. Let $0<q<1$. Let $F \in \mathcal{M}_{k}\left(\Gamma_{0}(N), \chi\right)$ and

$$
F(z)=\sum_{n \geq 0} a_{n} e^{2 \pi i n z} \quad(z \in \mathbb{H})
$$


be the Fourier expansion of $F$. Let $G:=F_{\mid \omega_{N}}$, where

$$
F_{\mid \omega_{N}}(z):=N^{-k / 2} z^{-k} F\left(-(N z)^{-1}\right)
$$

(which can be seen to belong to $\mathcal{M}_{k}\left(\Gamma_{0}(N), \bar{\chi}\right)$ ), and

$$
G(z)=\sum_{n \geq 0} b_{n} e^{2 \pi i n z} \quad(z \in \mathbb{H})
$$

be the Fourier expansion of $G$. Define $q$-series $\Lambda_{q}(s, F)$ and $\Lambda_{q}(s, G)$ by

$$
\begin{aligned}
& \Lambda_{q}(s, F):=(1-q)\left\{\sum_{n \in \mathbb{Z}} q^{n s}\left(f\left(\frac{q^{n}}{\sqrt{N}}\right)-a_{0}\right)-f\left(\frac{1}{\sqrt{N}}\right)\right\}, \\
& \Lambda_{q}(s, G):=(1-q)\left\{\sum_{n \in \mathbb{Z}} q^{n s}\left(g\left(\frac{q^{n}}{\sqrt{N}}\right)-b_{0}\right)-g\left(\frac{1}{\sqrt{N}}\right)\right\}
\end{aligned}
$$

for $\operatorname{Re}(s)>k$, where $f(y):=F(i y)$ and $g(y):=G(i y)$ for $y>0$. Then $\Lambda_{q}(s, F)$ and $\Lambda_{q}(s, G)$ can be analytically continued to meromorphic functions on $\mathbb{C}$,

$$
\frac{\Lambda_{q}(s, F)}{1-q}+\frac{a_{0}}{1-q^{s}}+\frac{b_{0} i^{k}}{1-q^{k-s}}
$$

and

$$
\frac{\Lambda_{q}(s, G)}{1-q}+\frac{b_{0}}{1-q^{s}}+\frac{a_{0} i^{-k}}{1-q^{k-s}}
$$

are entire, and they satisfy the functional equation

$$
\Lambda_{q}(s, F)=i^{k} \Lambda_{q}(k-s, G) .
$$

Proof. Since

$$
f\left(\frac{q^{n}}{\sqrt{N}}\right)=i^{k} q^{-n k} g\left(\frac{q^{-n}}{\sqrt{N}}\right) \quad(n \in \mathbb{Z}),
$$

we get

$$
\begin{aligned}
\frac{\Lambda_{q}(s, F)}{1-q}= & i^{k} \sum_{n \geq 1} q^{n(s-k)}\left(g\left(\frac{q^{-n}}{\sqrt{N}}\right)-b_{0}\right)+\sum_{n \geq 1} q^{-n s}\left(f\left(\frac{q^{-n}}{\sqrt{N}}\right)-a_{0}\right) \\
& -\frac{a_{0}}{1-q^{s}}-\frac{b_{0} i^{k}}{1-q^{k-s}}
\end{aligned}
$$

for $\operatorname{Re}(s)>k$. Similarly,

$$
\begin{aligned}
\frac{\Lambda_{q}(s, G)}{1-q}= & i^{-k} \sum_{n \geq 1} q^{n(s-k)}\left(f\left(\frac{q^{-n}}{\sqrt{N}}\right)-a_{0}\right)+\sum_{n \geq 1} q^{-n s}\left(g\left(\frac{q^{-n}}{\sqrt{N}}\right)-b_{0}\right) \\
& -\frac{b_{0}}{1-q^{s}}-\frac{a_{0} i^{-k}}{1-q^{k-s}}
\end{aligned}
$$


for $\operatorname{Re}(s)>k$. Since $f\left(q^{-n} / \sqrt{N}\right)-a_{0}=\sum_{m \geq 1} a_{m} e^{-2 \pi m q^{-n} / \sqrt{N}} \rightarrow 0$ and $g\left(q^{-n} / \sqrt{N}\right)-b_{0}=\sum_{m \geq 1} b_{m} e^{-2 \pi m q^{-n} / \sqrt{N}} \rightarrow 0$ rapidly as $n \rightarrow \infty$, all the series on the right-hand sides above converge absolutely and uniformly for $s$ in any compact subset of $\mathbb{C}$, whence they are entire. Hence $\Lambda_{q}(s, F)$ and $\Lambda_{q}(s, G)$ are meromorphic on $\mathbb{C}$, and the other claims follow immediately.

REMARK 2.2. When $q \rightarrow 1$,

$$
\Lambda_{q}(s, F) \rightarrow \Lambda(s, F):=\int_{0}^{\infty} y^{s-1}\left(F\left(\frac{i y}{\sqrt{N}}\right)-a_{0}\right) d y=\left(\frac{\sqrt{N}}{2 \pi}\right)^{s} \Gamma(s) L_{F}(s)
$$

for $\operatorname{Re}(s)>k$, where $L_{F}(s):=\sum_{n \geq 1} a_{n} n^{-s}(\operatorname{Re}(s)>k)$.

More generally, we get the following result for twisted automorphic forms, whose basic properties can be found in [1], [6].

Theorem 2.3. Let $0<q<1$ and $r \in \mathbb{N}$ with $(r, N)=1$. Let $F \in$ $\mathcal{M}_{k}\left(\Gamma_{0}(N), \chi\right)$ and $G:=F_{\mid \omega_{N}} \in \mathcal{M}_{k}\left(\Gamma_{0}(N), \bar{\chi}\right)$ have Fourier expansions (2.1), (2.2). Let $\psi$ be a primitive Dirichlet character modulo $r$ and $\tau(\psi):=$ $\sum_{m=1}^{r} \psi(m) e^{2 \pi i m / r}$ be the Gauss sum. Put $w(\psi):=\chi(r) \psi(N) \tau(\psi)^{2} r^{-1}$ and $M:=N r^{2}$. Let $F_{\psi}, G_{\psi}$ be the twisted series

$$
\begin{array}{rlrl}
F_{\psi}(z) & :=\sum_{n \geq 0} \psi(n) a_{n} e^{2 \pi i n z} & (z \in \mathbb{H}), \\
G_{\psi}(z) & :=\sum_{n \geq 0} \psi(n) b_{n} e^{2 \pi i n z} \quad(z \in \mathbb{H}) .
\end{array}
$$

Define $q$-series $\Lambda_{q}\left(s, F_{\psi}\right)$ and $\Lambda_{q}\left(s, G_{\psi}\right)$ by

$$
\begin{aligned}
& \Lambda_{q}\left(s, F_{\psi}\right):=(1-q)\left\{\sum_{n \in \mathbb{Z}} q^{n s}\left(f_{\psi}\left(\frac{q^{n}}{\sqrt{M}}\right)-\psi(0) a_{0}\right)-f_{\psi}\left(\frac{1}{\sqrt{M}}\right)\right\}, \\
& \Lambda_{q}\left(s, G_{\psi}\right):=(1-q)\left\{\sum_{n \in \mathbb{Z}} q^{n s}\left(g_{\psi}\left(\frac{q^{n}}{\sqrt{M}}\right)-\psi(0) b_{0}\right)-g_{\psi}\left(\frac{1}{\sqrt{M}}\right)\right\}
\end{aligned}
$$

for $\operatorname{Re}(s)>k$, where $f_{\psi}(y):=F_{\psi}(i y)$ and $g_{\psi}(y):=G_{\psi}(i y)$ for $y>0$. Then $\Lambda_{q}\left(s, F_{\psi}\right)$ and $\Lambda_{q}\left(s, G_{\psi}\right)$ can be analytically continued to meromorphic functions on $\mathbb{C}$,

$$
\frac{\Lambda_{q}\left(s, F_{\psi}\right)}{1-q}+\frac{\psi(0) a_{0}}{1-q^{s}}+\frac{w(\psi) \bar{\psi}(0) b_{0} i^{k}}{1-q^{k-s}}
$$

and

$$
\frac{\Lambda_{q}\left(s, G_{\psi}\right)}{1-q}+\frac{\psi(0) b_{0}}{1-q^{s}}+\frac{w(\bar{\psi})^{-1} \bar{\psi}(0) a_{0} i^{-k}}{1-q^{k-s}}
$$

are entire, and they satisfy the functional equation

$$
\Lambda_{q}\left(s, F_{\psi}\right)=i^{k} w(\psi) \Lambda_{q}\left(k-s, G_{\bar{\psi}}\right) .
$$


Proof. We can see that $F_{\psi} \in \mathcal{M}_{k}\left(\Gamma_{0}(M), \chi \psi^{2}\right), G_{\psi} \in \mathcal{M}_{k}\left(\Gamma_{0}(M), \bar{\chi} \psi^{2}\right)$ and $\left(F_{\psi}\right)_{\mid \omega_{M}}=w(\psi) G_{\bar{\psi}}$. In particular,

$$
f_{\psi}\left(q^{n} / \sqrt{M}\right)=w(\psi) i^{k} q^{-n k} g_{\bar{\psi}}\left(q^{-n} / \sqrt{M}\right) .
$$

Hence, as in the proof of Proposition 2.1, we get

$$
\begin{aligned}
\frac{\Lambda_{q}\left(s, F_{\psi}\right)}{1-q}= & w(\psi) i^{k} \sum_{n \geq 1} q^{n(s-k)}\left(g_{\bar{\psi}}\left(\frac{q^{-n}}{\sqrt{M}}\right)-\bar{\psi}(0) b_{0}\right) \\
& +\sum_{n \geq 1} q^{-n s}\left(f_{\psi}\left(\frac{q^{-n}}{\sqrt{M}}\right)-\psi(0) a_{0}\right)-\frac{\psi(0) a_{0}}{1-q^{s}}-\frac{w(\psi) \bar{\psi}(0) b_{0} i^{k}}{1-q^{k-s}}, \\
\frac{\Lambda_{q}\left(s, G_{\psi}\right)}{1-q}= & w(\bar{\psi})^{-1} i^{-k} \sum_{n \geq 1} q^{n(s-k)}\left(f_{\bar{\psi}}\left(\frac{q^{-n}}{\sqrt{M}}\right)-\bar{\psi}(0) a_{0}\right) \\
& +\sum_{n \geq 1} q^{-n s}\left(g_{\psi}\left(\frac{q^{-n}}{\sqrt{M}}\right)-\psi(0) b_{0}\right)-\frac{\psi(0) b_{0}}{1-q^{s}}-\frac{w(\bar{\psi})^{-1} \bar{\psi}(0) a_{0} i^{-k}}{1-q^{k-s}}
\end{aligned}
$$

for $\operatorname{Re}(s)>k$. This shows our claims.

REMARK 2.4. If $r \geq 2$, then $\psi(0)=0$, whence $\Lambda_{q}\left(s, F_{\psi}\right)$ and $\Lambda_{q}\left(s, G_{\psi}\right)$ are entire. If $r=1$, then $\psi=1$, whence Theorem 2.3 yields Proposition 2.1.

3. Converse theorems to Theorem 2.3. We show here converse theorems which are the main results of this paper. We begin with the simple case of $\Gamma_{0}(1)=\mathrm{SL}(2, \mathbb{Z})$. Actually, it corresponds to a corollary of Theorem 3.4.

3.1. The case of $\mathrm{SL}(2, \mathbb{Z})$

TheOREM 3.1. Let $k$ be a non-negative even integer. Suppose $F$ is given by the Fourier series

$$
F(z):=\sum_{n \geq 0} a_{n} e^{2 \pi i n z} \quad(z \in \mathbb{H})
$$

with $\left|a_{n}\right|=O\left(n^{\alpha}\right)$ as $n \rightarrow \infty$ for some $\alpha>0$. Let $f(y):=F($ iy $)$ for $y>0$. Let $\left\{q_{j}\right\}_{j=1}^{\infty} \subset(0,1)$ be a sequence which has a limit point in $(0,1]$. For any $q \in\left\{q_{j}\right\}_{j=1}^{\infty}$, assume the following:

(I) The function

$$
\Lambda_{q}(s, F):=(1-q)\left\{\sum_{n \in \mathbb{Z}} q^{n s}\left(f\left(q^{n}\right)-a_{0}\right)-f(1)\right\}
$$

can be analytically continued to a meromorphic function on $\mathbb{C}$ such that

$$
\frac{\Lambda_{q}(s, F)}{1-q}+a_{0}\left(\frac{1}{1-q^{s}}+\frac{i^{-k}}{1-q^{k-s}}\right)
$$

is entire. (We do not assume its boundedness in vertical strips.) 
(II) $\Lambda_{q}(s, F)$ satisfies the functional equation

$$
\Lambda_{q}(s, F)=i^{-k} \Lambda_{q}(k-s, F) .
$$

Then $F \in \mathcal{M}_{k}(\mathrm{SL}(2, \mathbb{Z}))$. In particular, if $a_{0}=0$, then $F \in \mathcal{S}_{k}(\mathrm{SL}(2, \mathbb{Z}))$, the space of cusp forms.

To prove this theorem, we need the following lemma, which gives an inversion formula for the $q$-Mellin transform.

Lemma 3.2. Let $0<q<1$. Let $f:(0, \infty) \rightarrow \mathbb{C}$ be a moderate asymptotic function of order $\left(i_{0}, j_{0}\right)$. Then

$$
\begin{aligned}
f\left(q^{n}\right) & =-\frac{\log q}{1-q} \cdot \frac{1}{2 \pi i} \int_{\sigma+i \pi / \log q}^{\sigma-i \pi / \log q} M_{q}(f)(s) q^{-n s} d s \\
& =-\frac{q^{-n \sigma} \log q}{2 \pi(1-q)} \int_{\pi / \log q}^{-\pi / \log q} M_{q}(f)(\sigma+i t) q^{-i n t} d t
\end{aligned}
$$

for $-i_{0}<\sigma<-j_{0}$ and $n \in \mathbb{Z}$.

Proof. Since for $-i_{0}<\sigma<-j_{0}$ and $t \in \mathbb{R}$ we have

$$
M_{q}(f)(\sigma+i t)=(1-q) \sum_{n \in \mathbb{Z}} q^{n(\sigma+i t)} f\left(q^{n}\right)=\sum_{n \in \mathbb{Z}}(1-q) q^{n \sigma} f\left(q^{n}\right) e^{2 \pi i n \cdot \frac{t \log q}{2 \pi}},
$$

we get

$$
\begin{aligned}
(1-q) q^{n \sigma} f\left(q^{n}\right) & =\int_{-1 / 2}^{1 / 2} M_{q}(f)\left(\sigma+i \cdot \frac{2 \pi t}{\log q}\right) e^{-2 \pi i n t} d t \\
& =-\frac{\log q}{2 \pi} \int_{\pi / \log q}^{-\pi / \log q} M_{q}(f)(\sigma+i t) q^{-i n t} d t
\end{aligned}
$$

for $-i_{0}<\sigma<-j_{0}$ and $n \in \mathbb{Z}$. Hence the lemma follows.

REMARK 3.3. A result similar to Lemma 3.2 is also given in $[2$, Theorem 2].

Proof of Theorem 3.1. Since $F(z)=\sum_{n \geq 0} a_{n} e^{2 \pi i n z}$, we have $F(z+1)=$ $F(z)$ for $z \in \mathbb{H}$. So it is sufficient to show that

$$
F\left(-z^{-1}\right)=z^{k} F(z)
$$

for $z \in \mathbb{H}$. Since $\left|a_{n}\right|=O\left(n^{\alpha}\right)$ as $n \rightarrow \infty$, the series $F(z)=\sum_{n \geq 0} a_{n} e^{2 \pi i n z}$ converges absolutely and uniformly for $z$ in any compact subset of $\mathbb{H}$, whence it is holomorphic on $\mathbb{H}$. So it is sufficient to show (3.2) for $z=i y$ with $y>0$ by analytic continuation. Note that $f(y)-a_{0}$ is a moderate asymptotic function of order $(-\alpha-1, *)$, where $*$ means any $j_{0} \in \mathbb{R}$ with $j_{0}<-\alpha-1$. 
Actually, it is clear that $f(y)-a_{0}=O\left(e^{-2 \pi y}\right)$ as $y \rightarrow \infty$. Also, since

$$
\Gamma(\alpha+1)=\lim _{n \rightarrow \infty} \frac{n^{\alpha} n !}{(\alpha+1) \cdots(\alpha+n)}=\lim _{n \rightarrow \infty} n^{\alpha} \cdot(-1)^{n}\left(\begin{array}{c}
-\alpha-1 \\
n
\end{array}\right)^{-1},
$$

we have

$$
\begin{aligned}
\left|f(y)-a_{0}\right| & \leq \sum_{n \geq 1}\left|a_{n}\right| e^{-2 \pi n y} \leq C_{\alpha} \sum_{n \geq 0}\left(\begin{array}{c}
-\alpha-1 \\
n
\end{array}\right)\left(-e^{-2 \pi y}\right)^{n} \\
& =C_{\alpha}\left(1-e^{-2 \pi y}\right)^{-\alpha-1}
\end{aligned}
$$

for some $C_{\alpha}>0$. This means that $f(y)-a_{0}=O\left(y^{-\alpha-1}\right)$ as $y \rightarrow 0$. Hence $f\left(q^{n}\right)-a_{0}=O\left(q^{-n(\alpha+1)}\right)$ as $n \rightarrow \infty$ and $f\left(q^{n}\right)-a_{0}=\sum_{m \geq 1} a_{m} e^{-2 \pi m q^{n}} \rightarrow 0$ rapidly as $n \rightarrow-\infty$. Therefore, the series $\sum_{n \in \mathbb{Z}} q^{n s}\left(f\left(q^{\bar{n}}\right)-a_{0}\right)$ converges absolutely on $\operatorname{Re}(s)>\alpha+1$. Put

$$
g(y):=f(y)-(i y)^{-k} f\left(y^{-1}\right)=F(i y)-(i y)^{-k} F\left(-(i y)^{-1}\right)
$$

for $y>0$. It is sufficient to show that $g(y) \equiv 0$ for $y>0$. We may assume that $\alpha>k-1$. We apply Lemma 3.2 to $q \in\left\{q_{j}\right\}_{j=1}^{\infty}$ and $f(y)-a_{0}$. By (I), (II), Cauchy's theorem and the $\frac{2 \pi i}{\log q} \mathbb{Z}$-periodicity of $\Lambda_{q}(s, F)$, for $n \in \mathbb{Z} \backslash\{0\}$ and a fixed $\sigma_{0}$ with $\sigma_{0}>\alpha+1$ we get

$$
\begin{aligned}
f\left(q^{n}\right)-a_{0}=-\frac{\log q}{1-q} \cdot \frac{1}{2 \pi i} \int_{\sigma_{0}+i \pi / \log q}^{\sigma_{0}-i \pi / \log q}\left(\Lambda_{q}(s, F)+(1-q) f(1)\right) q^{-n s} d s \\
=-\frac{\log q}{1-q} \cdot \frac{i^{-k}}{2 \pi i}\left\{\int_{k-\sigma_{0}+i \pi / \log q}^{k-\sigma_{0}-i \pi / \log q} \Lambda_{q}(k-s, F) q^{-n s} d s\right. \\
\quad+\int_{k-\sigma_{0}}^{\sigma_{0}} \Lambda_{q}\left(k-\sigma+i \frac{\pi}{\log q}, F\right) q^{-n(\sigma-i \pi / \log q)} d \sigma \\
\quad-\int_{k-\sigma_{0}}^{\sigma_{0}} \Lambda_{q}\left(k-\sigma-i \frac{\pi}{\log q}, F\right) q^{-n(\sigma+i \pi / \log q)} d \sigma \\
\left.\quad+2 \pi i\left(\operatorname{Res} \Lambda_{q}(k-s, F) q^{-n s}+\underset{s=k}{\operatorname{Res}} \Lambda_{q}(k-s, F) q^{-n s}\right)\right\} \\
=-\frac{\log q}{1-q} \cdot \frac{i^{-k}}{2 \pi i}\left\{\int_{\sigma_{0}+i \pi / \log q}^{\sigma_{0}-i \pi / \log q} \Lambda_{q}(s, F) q^{-n(k-s)} d s\right. \\
\quad+(-1)^{n} \int_{k-\sigma_{0}}^{\sigma_{0}}\left(\Lambda_{q}\left(k-\sigma+i \frac{\pi}{\log q}, F\right)-\Lambda_{q}\left(k-\sigma-i \frac{\pi}{\log q}, F\right)\right) q^{-n \sigma} d \sigma \\
\left.\quad+2 \pi i \cdot \frac{1-q}{\log q} a_{0}\left(i^{k}-q^{-n k}\right)\right\} \\
=\left(i q^{n}\right)^{-k}\left(f\left(q^{-n}\right)-a_{0}\right)+a_{0}\left(\left(i q^{n}\right)^{-k}-1\right)=\left(i q^{n}\right)^{-k} f\left(q^{-n}\right)-a_{0} .
\end{aligned}
$$


This means that $g\left(q^{n}\right)=0$ for $q \in\left\{q_{j}\right\}_{j=1}^{\infty}$ and $n \in \mathbb{Z} \backslash\{0\}$. In particular, as $n=1$, we have $g\left(q_{j}\right)=0(j=1,2, \ldots)$. Since $F$ is holomorphic on $\mathbb{H}$ and $f(y)=F(i y), f$ is holomorphic as a function on $\operatorname{Re}(y)>0$, and so is $g$. Hence we obtain $g(y) \equiv 0$ for $y>0$ by the unicity theorem. This completes the proof.

3.2. The case of $\Gamma_{0}(N)$

Theorem 3.4. Let $k, N \in \mathbb{N}$. Let $\omega_{N}:=\left(\begin{array}{cc}0 & -1 \\ N & 0\end{array}\right)$, and $\chi$ be a Dirichlet character modulo $N$ with $\chi( \pm 1)=( \pm 1)^{k}$. Suppose $F$ and $G$ are given by the Fourier series (2.1), (2.2) with $\left|a_{n}\right|,\left|b_{n}\right|=O\left(n^{\alpha}\right)$ as $n \rightarrow \infty$ for some $\alpha>0$. Let $f(y):=F(i y)$ and $g(y):=G(i y)$ for $y>0$. Let $\left\{q_{j}\right\}_{j=1}^{\infty} \subset(0,1)$ be a sequence which has a limit point in $(0,1]$. For any $q \in\left\{q_{j}\right\}_{j=1}^{\infty}$, assume that $\Lambda_{q}(s, F)$ and $\Lambda_{q}(s, G)$ defined by $(2.3),(2.4)$ can be analytically continued to meromorphic functions on $\mathbb{C}$ such that

$$
\frac{\Lambda_{q}(s, F)}{1-q}+\frac{a_{0}}{1-q^{s}}+\frac{b_{0} i^{k}}{1-q^{k-s}}
$$

and

$$
\frac{\Lambda_{q}(s, G)}{1-q}+\frac{b_{0}}{1-q^{s}}+\frac{a_{0} i^{-k}}{1-q^{k-s}}
$$

are entire, and satisfy the functional equation (2.5). Furthermore, put

$$
\mathcal{P}^{(N)}:=\bigcup_{\substack{a, c \in \mathbb{N} \\(a, c)=1}} \mathcal{P}_{a, c}^{(N)},
$$

where $\mathcal{P}_{a, c}^{(N)}:=\{p \in \mathbb{N} \mid p$ is a prime number such that $(p, N)=1, p \equiv a$ $(\bmod c)\}$. For any $p \in \mathcal{P}^{(N)}$ and any primitive Dirichlet character $\psi$ modulo $p$, put $M:=N p^{2}, \tau(\psi):=\sum_{m=1}^{p} \psi(m) e^{2 \pi i m / p}$ (the Gauss sum), and $w(\psi):=\chi(p) \psi(N) \tau(\psi)^{2} p^{-1}$, and let $F_{\psi}, G_{\psi}$ be the twisted series (2.7), (2.8). Let $f_{\psi}(y):=F_{\psi}(i y)$ and $g_{\psi}(y):=G_{\psi}(i y)$ for $y>0$. Let $\left\{q_{j}(\psi)\right\}_{j=1}^{\infty} \subset(0,1)$ be a sequence which has a limit point in $(0,1]$. For any $q \in\left\{q_{j}(\psi)\right\}_{j=1}^{\infty}$, assume that $\Lambda_{q}\left(s, F_{\psi}\right)$ and $\Lambda_{q}\left(s, G_{\psi}\right)$ defined by $(2.9),(2.10)$ can be analytically continued to entire functions and satisfy the functional equation (2.11). Then $F \in \mathcal{M}_{k}\left(\Gamma_{0}(N), \chi\right), G \in \mathcal{M}_{k}\left(\Gamma_{0}(N), \bar{\chi}\right)$ and $G=F_{\mid \omega_{N}}$.

We use the following lemma:

Lemma 3.5 (see [6, Lemma 7.10]). Let $p_{1}, p_{2}$ be prime numbers with $\left(p_{1} p_{2}, N\right)=1$. Put $M:=N p^{2}$ with $p=p_{1}$ or $p_{2}$. Suppose $F, G$ given by the Fourier series (2.1), (2.2) satisfy $G=F_{\mid \omega_{N}}$ and $\left(F_{\psi}\right)_{\mid \omega_{M}}=w(\psi) G_{\bar{\psi}}$ for any primitive Dirichlet character $\psi$ modulo $p$ with the constant $w(\psi)$ $:=\chi(p) \psi(N) \tau(\psi)^{2} p^{-1}$, where $F_{\psi}(z):=\sum_{n \geq 0} \psi(n) a_{n} e^{2 \pi i n z}$ and $G_{\bar{\psi}}(z):=$ $\sum_{n \geq 0} \bar{\psi}(n) b_{n} e^{2 \pi i n z}$ are the twisted series and $\tau(\psi):=\sum_{m=1}^{p} \psi(m) e^{2 \pi i m / p}$ is 
the Gauss sum. Then

$$
G_{\mid \gamma}=\bar{\chi}\left(p_{2}\right) G \quad \text { for any } \gamma=\left(\begin{array}{rr}
p_{1} & v \\
u N & p_{2}
\end{array}\right) \in \Gamma_{0}(N)(u, v \in \mathbb{Z}),
$$

where

$$
G_{\mid \gamma}(z):=(c z+d)^{-k} G\left(\frac{a z+b}{c z+d}\right) \quad \text { if } \gamma=\left(\begin{array}{cc}
a & b \\
c & d
\end{array}\right) \in \Gamma_{0}(N) .
$$

Proof of Theorem 3.4. First we show that $G=F_{\mid \omega_{N}}$. Note that $f(y)-a_{0}$ and $g(y)-b_{0}$ are moderate asymptotic functions of order $(-\alpha-1, *)$. We may assume that $\alpha>k-1$. It is easy to see that $F$ and $G$ are holomorphic on $\mathbb{H}$, and the series $\sum_{n \in \mathbb{Z}} q^{n s}\left(f\left(q^{n} / \sqrt{N}\right)-a_{0}\right)$ and $\sum_{n \in \mathbb{Z}} q^{n s}\left(g\left(q^{n} / \sqrt{N}\right)-b_{0}\right)$ converge absolutely for $\operatorname{Re}(s)>\alpha+1$. Put

$h(y):=f\left(\frac{y}{\sqrt{N}}\right)-i^{k} y^{-k} g\left(\frac{1}{\sqrt{N} y}\right)=i^{k} y^{-k}\left\{F_{\mid \omega_{N}}\left(\frac{i}{\sqrt{N} y}\right)-G\left(\frac{i}{\sqrt{N} y}\right)\right\}$

for $y>0$. It is sufficient to show that $h(y) \equiv 0$ for $y>0$. By applying Lemma 3.2 to $q \in\left\{q_{j}\right\}_{j=1}^{\infty}, f(y)-a_{0}$ and $g(y)-b_{0}$ and imitating the proof of Theorem 3.1, we obtain $h\left(q^{n}\right)=0$ for all $q \in\left\{q_{j}\right\}_{j=1}^{\infty}$ and $n \in \mathbb{Z} \backslash\{0\}$. In particular, as $n=1$, we have $h\left(q_{j}\right)=0(j=1,2, \ldots)$. Hence $h(y) \equiv 0$ for $y>0$ by holomorphy of $F, G$ on $\mathbb{H}$ and the unicity theorem.

Next we show the automorphy of $F, G$ by using Lemma 3.5. We have $G=$ $F_{\mid \omega_{N}}$ (which is equivalent to $F=(-1)^{k} G_{\mid \omega_{N}}$ ). Moreover, by considering $F_{\psi}$, $w(\psi) G_{\bar{\psi}}$ and $M$ instead of $F, G$ and $N$ respectively, we also get $\left(F_{\psi}\right)_{\mid \omega_{M}}=$ $w(\psi) G_{\bar{\psi}}$. So it is sufficient to show the automorphy of either $F$ or $G$. We show the latter, i.e.,

$$
G_{\mid \gamma}=\bar{\chi}(d) G \quad \text { if } \gamma=\left(\begin{array}{ll}
a & b \\
c & d
\end{array}\right) \in \Gamma_{0}(N) .
$$

When $c=0$, since $G(z+1)=G(z)$ for $z \in \mathbb{H}$ by the Fourier series (2.2), we get

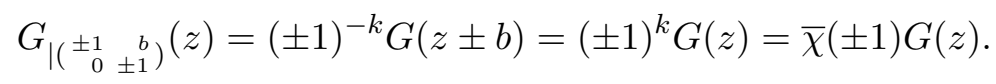

When $c \neq 0$, we have $|c| \in \mathbb{N}, c \equiv 0(\bmod N)$ and $(a d, c)=1$. If $G_{\mid\left(\begin{array}{ll}a & b \\ c & d\end{array}\right)}=$ $\bar{\chi}(d) G$, then

$$
G_{\mid\left(\begin{array}{ll}
-a & -b \\
-c & -d
\end{array}\right)}=(-1)^{k} G_{\mid\left(\begin{array}{ll}
a & b \\
c & d
\end{array}\right)}=\bar{\chi}(-1) \bar{\chi}(d) G=\bar{\chi}(-d) G .
$$

So we may assume that $c>0$. Note that for any $a, c \in \mathbb{N}$ with $(a, c)=1, \mathcal{P}_{a, c}^{(N)}$ is a countably infinite set (by Dirichlet's theorem on primes in an arithmetic progression). Hence, choose $m_{1}, m_{2} \in \mathbb{N} \cup\{0\}$ such that $a+c m_{1}, d+c m_{2}>0$, and prime numbers $p_{1}, p_{2}$ such that $p_{1} \in \mathcal{P}_{a+c m_{1}, c}^{(N)}, p_{2} \in \mathcal{P}_{d+c m_{2}, c}^{(N)}$. This 
means that $p_{1}, p_{2}$ can be written as $p_{1}=a+c t_{1}, p_{2}=d+c t_{2}\left(t_{1}, t_{2} \in \mathbb{Z}\right)$. Then

$$
\gamma=\left(\begin{array}{ll}
a & b \\
c & d
\end{array}\right)=\left(\begin{array}{rr}
1 & -t_{1} \\
0 & 1
\end{array}\right)\left(\begin{array}{rr}
p_{1} & v \\
u N & p_{2}
\end{array}\right)\left(\begin{array}{rr}
1 & -t_{2} \\
0 & 1
\end{array}\right)
$$

with $u:=c / N \in \mathbb{Z}$ and $v:=b+p_{1} t_{2}+p_{2} t_{1}-c t_{1} t_{2} \in \mathbb{Z}$. Hence, by Lemma 3.5,

$$
\begin{aligned}
& G_{\mid \gamma}=G_{\mid\left(\begin{array}{ll}
1 & -t_{1} \\
0 & 1
\end{array}\right)\left(\begin{array}{cc}
p_{1} & v \\
u N & p_{2}
\end{array}\right)\left(\begin{array}{cc}
1 & -t_{2} \\
0 & 1
\end{array}\right)}=\bar{\chi}(1) G_{\mid\left(\begin{array}{cc}
p_{1} & v \\
u N & p_{2}
\end{array}\right)\left(\begin{array}{ll}
1 & -t_{2} \\
0 & 1
\end{array}\right)} \\
& =1 \cdot \bar{\chi}\left(p_{2}\right) G_{\mid\left(\begin{array}{ll}
1 & -t_{2} \\
0 & 1
\end{array}\right)}=\bar{\chi}\left(p_{2}-t_{2} u N\right) \cdot \bar{\chi}(1) G=\bar{\chi}(d) G \text {. }
\end{aligned}
$$

\section{An application to the Jacobi theta function}

4.1. A q-analogue $\zeta^{(q)}(s)$ of the Riemann zeta function. We recall the $q$-analogue of the complete Riemann zeta function $\zeta^{(q)}(s)$ introduced in [3] and [4]. Let $0<q<1$. Put $\theta(y):=\vartheta(i y)=\sum_{m \in \mathbb{Z}} e^{-\pi m^{2} y}(y>0)$ and $\phi(y):=\theta\left(y^{2}\right)(y>0)$, where $\vartheta(z):=\sum_{m \in \mathbb{Z}} e^{\pi i m^{2} z}(z \in \mathbb{H})$ is the Jacobi theta function. Since $\theta\left(y^{-1}\right)=\sqrt{y} \theta(y), \phi(y)-1$ is a moderate asymptotic function of order $(-1, *)\left(\phi(y)-1=O\left(e^{-\pi y^{2}}\right)\right.$ as $\left.y \rightarrow \infty\right)$. Then $\zeta^{(q)}(s)$ can be obtained by the $q$-Mellin transform of $\phi(y)-1$, i.e.,

$$
\begin{aligned}
\zeta^{(q)}(s) & :=M_{q}(\phi-1)(s)=(1-q) \sum_{n \in \mathbb{Z}} q^{n s}\left(\phi\left(q^{n}\right)-1\right) \\
& =(1-q) \sum_{n \in \mathbb{Z}} q^{n s} \sum_{m \neq 0} e^{-\pi m^{2} q^{2 n}}
\end{aligned}
$$

for $\operatorname{Re}(s)>1$. Note that $\zeta^{(q)}(s)$ tends to the complete Riemann zeta function as $q \rightarrow 1$, i.e.,

$$
\zeta^{(q)}(s) \rightarrow \int_{0}^{\infty} y^{s-1}\left(\theta\left(y^{2}\right)-1\right) d y=\pi^{-s / 2} \Gamma\left(\frac{s}{2}\right) \zeta(s) \quad(\operatorname{Re}(s)>1) .
$$

Since

$$
\begin{aligned}
\frac{\zeta^{(q)}(s)}{1-q}= & \sum_{m \in \mathbb{Z}} e^{-\pi m^{2}}+\sum_{n \geq 1}\left(q^{-n s}+q^{-n(1-s)}\right) \sum_{m \neq 0} e^{-\pi m^{2} q^{-2 n}} \\
& -\frac{1}{1-q^{s}}-\frac{1}{1-q^{1-s}},
\end{aligned}
$$

$\zeta^{(q)}(s)$ can be analytically continued to a meromorphic function on $\mathbb{C}$. More precisely,

$$
\frac{\zeta^{(q)}(s)}{1-q}+\frac{1}{1-q^{s}}+\frac{1}{1-q^{1-s}}
$$

is entire and satisfies the functional equation

$$
\zeta^{(q)}(1-s)=\zeta^{(q)}(s)
$$


4.2. A characterization of $q$-Mellin's image of the theta function. In this subsection, we show that the only moderate asymptotic function which gives the meromorphy as in (4.1) and the functional equation (4.2) under some reasonable conditions is given by $\theta\left(y^{2}\right)-1(y>0)$. The idea of the proof is the same as for Theorem 3.1. Note that, in the classical case, we have a similar description of the Riemann zeta function (due to Hamburger, see [8]).

TheOREM 4.1. Let

$$
F(z):=\sum_{n \geq 0} a_{n} e^{\pi i n^{2} z} \quad(z \in \mathbb{H})
$$

with $\left|a_{n}\right|=O\left(n^{\beta}\right)$ as $n \rightarrow \infty$ for some $0<\beta<1$. Let $f(y):=F($ iy $)$ for $y>0$. Let $\left\{q_{j}\right\}_{j=1}^{\infty} \subset(0,1)$ be a sequence which has a limit point in $(0,1]$. For any $q \in\left\{q_{j}\right\}_{j=1}^{\infty}$, assume the following:

(I) The function

$$
L_{q}(f)(s):=(1-q) \sum_{n \in \mathbb{Z}} q^{n s}\left(f\left(q^{2 n}\right)-a_{0}\right)
$$

can be analytically continued to a meromorphic function on $\mathbb{C}$ such that

$$
\frac{L_{q}(f)(s)}{1-q}+\frac{1}{1-q^{s}}+\frac{1}{1-q^{1-s}}
$$

is entire.

(II) $L_{q}(f)(s)$ satisfies the functional equation

$$
L_{q}(f)(1-s)=L_{q}(f)(s) .
$$

Then $a_{n}=2$ for $n=1,2, \ldots$ In particular, if $a_{0}=1$, then $F(z)=\vartheta(z)$ and $f(y)=\theta(y)$. In other words, $L_{q}(f)(s)$ agrees essentially with $\zeta^{(q)}(s)$.

Proof. The function $f\left(y^{2}\right)-a_{0}$ is a moderate asymptotic function of order $(-2(\beta+1), *)$. By applying Lemma 3.2 to $q \in\left\{q_{j}\right\}_{j=1}^{\infty}, f\left(y^{2}\right)-a_{0}$ and $\sigma>2(\beta+1)$ and imitating the proof of Theorem 3.1, we have

$$
f\left(q^{2 n}\right)-a_{0}=q^{-n}\left(f\left(q^{-2 n}\right)-a_{0}\right)+q^{-n}-1 \quad(n \in \mathbb{Z})
$$

for $q \in\left\{q_{j}\right\}_{j=1}^{\infty}$. Hence

$$
f(y)-a_{0}=\frac{1}{\sqrt{y}}\left(f\left(y^{-1}\right)-a_{0}\right)+\frac{1}{\sqrt{y}}-1 \quad(y>0)
$$

by the holomorphy of $F$ on $\mathbb{H}$ and the unicity theorem. We multiply this by $e^{-\pi t^{2} y}(t>0)$ and integrate over $y \in(0, \infty)$. From the left-hand side of (4.4), we get

$$
\int_{0}^{\infty}\left(f(y)-a_{0}\right) e^{-\pi t^{2} y} d y=\sum_{n \geq 1} \frac{a_{n}}{\pi\left(t^{2}+n^{2}\right)}
$$


for $t>0$, where the series on the right-hand side converges absolutely since $\left|a_{n}\right|=O\left(n^{\beta}\right)$ as $n \rightarrow \infty(\beta<1)$. From the right-hand side of (4.4), we get

$$
\int_{0}^{\infty}\left\{\frac{1}{\sqrt{y}}\left(f\left(y^{-1}\right)-a_{0}\right)+\frac{1}{\sqrt{y}}-1\right\} e^{-\pi t^{2} y} d y=\frac{1}{t} \sum_{n \geq 1} a_{n} e^{-2 \pi n t}+\frac{1}{t}-\frac{1}{\pi t^{2}}
$$

for $t>0$. Hence

$$
\sum_{n \geq 1} a_{n}\left(\frac{1}{t+i n}+\frac{1}{t-i n}\right)-2 \pi+\frac{2}{t}=2 \pi \sum_{n \geq 1} a_{n} e^{-2 \pi n t} \quad(t>0) .
$$

The series on the left-hand side is a meromorphic function on $\mathbb{C}$ and has simple poles at $t= \pm i n$ with residues $a_{n}(n=1,2, \ldots)$. Since the series on the right-hand side is an $i \mathbb{Z}$-periodic function, so is the function on the left-hand side. In particular, all the residues at $t= \pm$ in $(n=1,2, \ldots)$ are equal to the residue at $t=0$. Hence $a_{n}=2$ for $n=1,2, \ldots$

4.3. Remarks on a q-Mellin image. From the previous study, one finds that the essential problem is whether (4.4) follows from (4.3) with a fixed $q \in(0,1)$.

Let us consider the following subset of $(0,1)$ :

$$
\mathcal{Y}:=\bigcap_{j \geq 0} \bigcup_{n \geq 1} Y_{j, n}
$$

where

$$
Y_{j, n}:=\bigcup_{\substack{m_{1}, m_{2} \geq 0 \\ m_{1} \neq m_{2}}}\left\{y \in(0,1) \mid\left(\pi n y^{2 m_{1}}, \pi n y^{2 m_{2}}\right) \in X_{j}\right\} \quad(j \in \mathbb{N} \cup\{0\}, n \in \mathbb{N})
$$

and

$X_{j}:=\bigcap_{a_{1}, a_{2} \geq 0}\left\{\left(x_{1}, x_{2}\right) \in \mathbb{R}_{>0}^{2} \mid e^{2 j x_{1}} \cosh \left(\left(2 a_{1}+1\right) x_{1}\right) \neq e^{2 j x_{2}} \cosh \left(\left(2 a_{2}+1\right) x_{2}\right)\right\}$ $(j \in \mathbb{N} \cup\{0\})$ with $\mathbb{R}_{>0}^{2}:=\left\{\left(x_{1}, x_{2}\right) \in \mathbb{R}^{2} \mid x_{1}, x_{2}>0\right\}$. The set $\mathcal{Y}$ plays an important role in Lemma 4.6 below. We have the following proposition about $\mathcal{Y}$.

Proposition 4.2. The set $\mathcal{Y}$ defined by (4.5) has infinitely many elements.

To prove this, we first show the following lemma.

Lemma 4.3. There does not exist any $(a, b, c, d) \in \mathbb{Z}^{4}$ such that

$$
2 \leq \frac{e^{a \pi / 2}+e^{b \pi / 2}}{e^{c \pi / 2}+e^{d \pi / 2}} \leq \frac{1}{2} e^{\pi / 2}
$$


Proof. Suppose that $a, b, c, d \in \mathbb{R}$ satisfy these inequalities. We may assume that $a \geq b$ and $c \geq d$. Then

$$
2 \leq \frac{e^{a \pi / 2}+e^{b \pi / 2}}{e^{c \pi / 2}+e^{d \pi / 2}}<\frac{e^{a \pi / 2}+e^{b \pi / 2}}{e^{c \pi / 2}}=e^{(a-c) \pi / 2}+e^{(b-c) \pi / 2} \leq 2 e^{(a-c) \pi / 2} .
$$

It follows that $c<a$. Also,

$$
\begin{aligned}
e^{a \pi / 2} & <e^{a \pi / 2}+e^{b \pi / 2} \leq \frac{1}{2} e^{\pi / 2}\left(e^{c \pi / 2}+e^{d \pi / 2}\right) \leq \frac{1}{2} e^{\pi / 2}\left(e^{c \pi / 2}+e^{c \pi / 2}\right) \\
& =e^{(c+1) \pi / 2}
\end{aligned}
$$

Hence $a<c+1$. Thus, $c<a<c+1$, so that $(a, c) \notin \mathbb{Z}^{2}$.

Proof of Proposition 4.2. From Lemma 4.3, it follows that

$$
\begin{aligned}
& e^{2 \pi j} \cosh \left(\left(2 a_{1}+1\right) \pi\right) \neq e^{\pi j / 2} \cosh \left(\left(2 a_{2}+1\right) \frac{\pi}{4}\right) \\
& \left(j, a_{1}, a_{2} \in \mathbb{N} \cup\{0\}\right) .
\end{aligned}
$$

Actually, $2<e^{\pi / 4}<e^{\pi / 2} / 2$ and (4.6) is equivalent to

$$
\frac{e^{\left(4 a_{1}+2+3 j\right) \pi / 2}+e^{-\left(4 a_{1}+2-3 j\right) \pi / 2}}{e^{a_{2} \pi / 2}+e^{-\left(a_{2}+1\right) \pi / 2}} \neq e^{\pi / 4} \quad\left(j, a_{1}, a_{2} \in \mathbb{N} \cup\{0\}\right) .
$$

Hence $(\pi, \pi / 4) \in X_{j}$ for $j \in \mathbb{N} \cup\{0\}$. Thus,

$$
\left\{2^{-1}, 2^{-1 / 2}, 2^{-1 / 3}, \ldots\right\} \subset Y_{j, 1}
$$

for all $j \in \mathbb{N} \cup\{0\}$. Therefore,

$$
\mathcal{Y}=\bigcap_{j \geq 0} \bigcup_{n \geq 1} Y_{j, n} \supset\left\{2^{-1}, 2^{-1 / 2}, 2^{-1 / 3}, \ldots\right\} .
$$

REMARK 4.4. Since the function $\cosh (y)=\left(e^{y}+e^{-y}\right) / 2$ is increasing for $y>0$, we have

$$
X_{0}=\bigcap_{a_{1}, a_{2} \geq 0}\left\{\left(x_{1}, x_{2}\right) \in \mathbb{R}_{>0}^{2} \mid x_{1} \neq \frac{2 a_{2}+1}{2 a_{1}+1} x_{2}\right\},
$$

and therefore

$$
Y_{0, n}=\bigcup_{m \geq 1} \bigcap_{a_{1}, a_{2} \geq 0}\left\{y \in(0,1) \mid y \neq\left(\frac{2 a_{2}+1}{2 a_{1}+1}\right)^{1 / 2 m}\right\} \quad(n \in \mathbb{N}),
$$

which does not depend on $n$. Hence

$$
\mathcal{Y} \subset \bigcup_{n \geq 1} Y_{0, n}=\bigcup_{m \geq 1} \bigcap_{a_{1}, a_{2} \geq 0}\left\{y \in(0,1) \mid y \neq\left(\frac{2 a_{2}+1}{2 a_{1}+1}\right)^{1 / 2 m}\right\} .
$$

Therefore, for example, $3^{-1 / 2}, 5^{-1 / 2}, 7^{-1 / 2}, \ldots \notin \mathcal{Y}$.

Now we give a characterization for a fixed $q$. 
Theorem 4.5. Let $F(z):=\sum_{n>0} a_{n} e^{\pi i n^{2} z}(z \in \mathbb{H})$ with $\left|a_{n}\right|=O\left(n^{\beta}\right)$ as $n \rightarrow \infty$ for some $\beta>0$. Let $f(y):=F($ iy $)$ for $y>0$. Take $q \in(0,1)$. For this q, assume (I) and (II) in Theorem 4.1 hold. Then

$$
f\left(q^{2 n}\right)-a_{0}=q^{-n}\left(f\left(q^{-2 n}\right)-a_{0}\right)+q^{-n}-1 \quad(n \in \mathbb{Z}) .
$$

If $q \in \mathcal{Y}$ and $f\left(q^{2 m}\right)=\theta\left(q^{2 m}\right)(m=0,1, \ldots)$, then $f(y)=\theta(y)(y>0)$, whence $F(z)=\vartheta(z)(z \in \mathbb{H})$.

To prove this theorem, we need the following lemma.

Lemma 4.6. Let $F(z):=\sum_{n \geq 0} a_{n} e^{\pi i n^{2} z}$ and $G(z):=\sum_{n \geq 0} b_{n} e^{\pi i n^{2} z}$ converge absolutely for $z \in \mathbb{H}$. Take $q \in \mathcal{Y}$. Assume that $F\left(i q^{2 m}\right)=G\left(i q^{2 m}\right)$ for $m=0,1, \ldots$ Then $a_{n}=b_{n}$ for $n=0,1, \ldots$, i.e., $F(z) \equiv G(z)(z \in \mathbb{H})$.

Proof. Put

$$
A:=\left\{\left(c_{0}, c_{1}, \ldots\right) \in \prod_{n \geq 0} \mathbb{C} \mid \sum_{n \geq 0} c_{n} e^{-\pi n^{2} q^{2 m}}=0 \text { for } m=0,1, \ldots\right\} .
$$

It is sufficient to show that $A=\{(0,0, \ldots)\}$. It is clear that $(0,0, \ldots) \in A$. For $m \in \mathbb{N} \cup\{0\}$, put

$$
A_{m}:=\left\{\left(c_{0 m}, c_{1 m}, \ldots\right) \in \prod_{n \geq 0} \mathbb{C} \mid \sum_{n \geq 0} c_{n m} e^{-\pi n^{2} q^{2 m}}=0\right\} .
$$

Note that $A=\bigcap_{m \geq 0} A_{m}$. It is well known that the (general) theta function

$$
\vartheta(z, \tau):=\sum_{n \in \mathbb{Z}} e^{\pi i n^{2} \tau+2 \pi i n z}
$$

converges absolutely for $(z, \tau) \in \mathbb{C} \times \mathbb{H}$ and its zeros are $\{z \in \mathbb{C} \mid \vartheta(z, \tau)=0\}$ $=\{(a+1 / 2) \tau+(b+1 / 2) \mid a, b \in \mathbb{Z}\}$ for $\tau \in \mathbb{H}$, by the Jacobi triple product formula

$$
\vartheta(z, \tau)=\prod_{n \geq 1}\left(1-e^{2 \pi i n \tau}\right)\left(1+e^{(2 n-1) \pi i \tau+2 \pi i z}\right)\left(1+e^{(2 n-1) \pi i \tau-2 \pi i z}\right) .
$$

In particular, when $\tau=i q^{2 m}(m \in \mathbb{N} \cup\{0\})$, the zeros of $\vartheta\left(z, i q^{2 m}\right)=$ $1+2 \sum_{n \geq 1} \cos (2 \pi n z) e^{-\pi n^{2} q^{2 m}}$ are $z=(a+1 / 2) i q^{2 m}+(b+1 / 2)(a, b \in \mathbb{Z})$. This means that

$$
\begin{aligned}
& \left\{\left(c_{1 m}, c_{2 m}, \ldots\right) \in \prod_{n \geq 1} \mathbb{C} \mid 1+\sum_{n \geq 1} c_{n m} e^{-\pi n^{2} q^{2 m}}=0\right\} \\
= & \bigcup_{a \geq 0}\left\{\left(c_{1 m}, c_{2 m}, \ldots\right) \in \prod_{n \geq 1} \mathbb{C} \mid c_{n m}=2(-1)^{n} \cosh \left(2 \pi n\left(a+\frac{1}{2}\right) q^{2 m}\right)\right\}
\end{aligned}
$$

because the maps $\mathbb{C} \ni z \mapsto 2 \cos (2 \pi n z) \in \mathbb{C}(n=1,2, \ldots)$ are surjective. Now fix $m \in \mathbb{N} \cup\{0\}$ and suppose that $\sum_{n \geq 0} c_{n m} e^{-\pi n^{2} q^{2 m}}=0$ with 
$\left(c_{0 m}, c_{1 m}, \ldots\right) \neq(0,0, \ldots)$. We put $j:=\min \left\{n \in \mathbb{N} \cup\{0\} \mid c_{n m} \neq 0\right\}$. Then $c_{0 m}=\cdots=c_{j-1, m}=0$ and

$$
\begin{aligned}
0 & =\sum_{n \geq j} c_{n m} e^{-\pi n^{2} q^{2 m}}=\sum_{n \geq 0} c_{n+j, m} e^{-\pi(n+j)^{2} q^{2 m}} \\
& =c_{j m} e^{-\pi j^{2} q^{2 m}} \sum_{n \geq 0} \frac{c_{n+j, m}}{c_{j m}} e^{-2 \pi j n q^{2 m}} e^{-\pi n^{2} q^{2 m}} .
\end{aligned}
$$

Hence

$$
1+\sum_{n \geq 1} \frac{c_{n+j, m}}{c_{j m}} e^{-2 \pi j n q^{2 m}} \cdot e^{-\pi n^{2} q^{2 m}}=0 .
$$

From (4.7), it follows that

$$
\begin{array}{r}
c_{n+j, m} \in\left\{c_{j m} e^{2 \pi j n q^{2 m}} \cdot 2(-1)^{n} \cosh \left(2 \pi n(a+1 / 2) q^{2 m}\right) \mid a \in \mathbb{N} \cup\{0\}\right\} \\
(n=1,2, \ldots) .
\end{array}
$$

Therefore,

$$
A_{m}=\{(0,0, \ldots)\} \cup \bigcup_{a, j \geq 0} B_{m, a}^{j} \quad(m \in \mathbb{N} \cup\{0\}),
$$

where

$$
\begin{aligned}
B_{m, a}^{j} & :=\left\{\left(c_{0 m}, c_{1 m}, \ldots\right) \in \prod_{n \geq 0} \mathbb{C} \mid c_{0 m}=\cdots=c_{j-1, m}=0, c_{j m} \neq 0,\right. \\
& \left.c_{n+j, m}=c_{j m} e^{2 \pi j n q^{2 m}} \cdot 2(-1)^{n} \cosh \left(2 \pi n(a+1 / 2) q^{2 m}\right)(n=1,2, \ldots)\right\} .
\end{aligned}
$$

It is sufficient to show that $\bigcap_{m \geq 0} \bigcup_{a, j \geq 0} B_{m, a}^{j}=\emptyset$. It is clear that $B_{m_{1}, a_{1}}^{j_{1}} \cap$ $B_{m_{2}, a_{2}}^{j_{2}}=\emptyset$ for any $m_{1}, m_{2}, a_{1}, a_{2}, j_{1}, j_{2} \in \mathbb{N} \cup\{0\}$ with $j_{1} \neq j_{2}$. Hence, it is sufficient to show that

$$
\bigcap_{m \geq 0} \bigcup_{a \geq 0} B_{m, a}^{j}=\emptyset \quad(j=0,1, \ldots) .
$$

Note that (4.8) is equivalent to

$$
\bigcap_{m \geq 0} \bigcup_{a_{0}, \ldots, a_{m} \geq 0}\left(B_{0, a_{0}}^{j} \cap \cdots \cap B_{m, a_{m}}^{j}\right)=\emptyset \quad(j=0,1, \ldots) .
$$

This means that for $j=0,1, \ldots$, there exists $m=m(j) \in \mathbb{N} \cup\{0\}$ such that $B_{0, a_{0}}^{j} \cap \cdots \cap B_{m, a_{m}}^{j}=\emptyset$ for any $a_{0}, \ldots, a_{m} \in \mathbb{N} \cup\{0\}$. Therefore, it is sufficient to show that for $j=0,1, \ldots$, there exist $m_{1}, m_{2} \in \mathbb{N} \cup\{0\}\left(m_{1} \neq m_{2}\right)$ such that $B_{m_{1}, a_{1}}^{j} \cap B_{m_{2}, a_{2}}^{j}=\emptyset$ for any $a_{1}, a_{2} \in \mathbb{N} \cup\{0\}$. We have the following expression: 


$$
\begin{gathered}
B_{m_{1}, a_{1}}^{j} \cap B_{m_{2}, a_{2}}^{j}=\left\{\left(c_{0}, c_{1}, \ldots\right) \in \prod_{n \geq 0} \mathbb{C} \mid c_{0}=\cdots=c_{j-1}=0, c_{j} \neq 0,\right. \\
c_{n+j}=c_{j} e^{2 \pi j n q^{2 m_{1}}} \cdot 2(-1)^{n} \cosh \left(\pi n\left(2 a_{1}+1\right) q^{2 m_{1}}\right) \\
\left.=c_{j} e^{2 \pi j n q^{2 m_{2}}} \cdot 2(-1)^{n} \cosh \left(\pi n\left(2 a_{2}+1\right) q^{2 m_{2}}\right)(n=1,2, \ldots)\right\} .
\end{gathered}
$$

Hence the assertion follows immediately from the definition of $\mathcal{Y}$.

REMARK 4.7. Since the sequence $\left\{i q^{2 m}\right\}_{m=0}^{\infty}$ does not have any limit points in $\mathbb{H}$, we cannot use the unicity theorem.

Proof of Theorem 4.5. The first statement follows as in the proof of Theorem 3.1. Note that $\theta(y)=1+2 \sum_{m \geq 1} e^{-\pi m^{2} y}$ satisfies

$$
\theta(y)-1=\frac{1}{\sqrt{y}}\left(\theta\left(y^{-1}\right)-1\right)+\frac{1}{\sqrt{y}}-1 \quad \text { for } y>0
$$

In particular,

$$
\theta\left(q^{2 n}\right)-1=q^{-n}\left(\theta\left(q^{-2 n}\right)-1\right)+q^{-n}-1 \quad(n \in \mathbb{Z}) .
$$

Hence, if $q \in \mathcal{Y}$ and $f\left(q^{2 m}\right)=\theta\left(q^{2 m}\right)(m=0,1,2, \ldots)$, then $a_{0}=1$ and $f(y)=\theta(y)(y>0)$ by Lemma 4.6.

Finally, we obtained two characterizations, in Theorems 4.1 and 4.5. Note that Theorem 4.1 requires a strong assumption on $q$, and Theorem 4.5 on $f$. It seems difficult to combine the two theorems.

A. Appendix - An inversion formula. In this appendix, we show an inversion formula which is different from (3.1). It gives a generalization of the classical Mellin inversion formula in terms of the $q$-Mellin transform.

Proposition A.1. Let $0<q<1$. Let $f:(0, \infty) \rightarrow \mathbb{C}$ be a moderate asymptotic function of order $\left(i_{0}, j_{0}\right)$. Put

$$
M_{q}^{N}(f)(s):=-\frac{1-q}{\log q} \sum_{|n| \leq N} M(f)\left(s+\frac{2 \pi i n}{\log q}\right)
$$

for $-i_{0}<\operatorname{Re}(s)<-j_{0}$ and $N \in \mathbb{N} \cup\{0\}$, where $M(f)(s)=\int_{0}^{\infty} y^{s-1} f(y) d y$. Fix $\sigma$ with $-i_{0}<\sigma<-j_{0}$. Then

$$
\begin{aligned}
& \text { (A.1) } f(y) \\
& =\left\{\begin{array}{l}
-\frac{\log q}{1-q} \cdot \frac{1}{2 N+1} \cdot \frac{1}{2 \pi i} \int_{\sigma-i \infty}^{\sigma+i \infty} M_{q}^{N}(f)(s) y^{-s} d s \quad \text { if } y=q^{n}(n \in \mathbb{Z}), \\
-\frac{\log q}{1-q} \cdot \frac{\sin \left(\pi \frac{\log y}{\log q}\right)}{\sin \left((2 N+1) \pi \frac{\log y}{\log q}\right)} \cdot \frac{1}{2 \pi i} \int_{\sigma-i \infty}^{\sigma+i \infty} M_{q}^{N}(f)(s) y^{-s} d s \\
\text { if } y \neq q^{n /(2 N+1)}(n \in \mathbb{Z}),
\end{array}\right.
\end{aligned}
$$


for $y>0$ and $N \in \mathbb{N} \cup\{0\}$. Moreover, if the sum $M_{q}^{N}(f)(\sigma+i t)$ converges absolutely and uniformly for $t$ in any compact subset of $\mathbb{R}$ as $N \rightarrow \infty$, then

$$
\lim _{N \rightarrow \infty} M_{q}^{N}(f)(\sigma+i t)=M_{q}(f)(\sigma+i t) \quad(t \in \mathbb{R}),
$$

where $M_{q}(f)(s)=(1-q) \sum_{n \in \mathbb{Z}} q^{n s} f\left(q^{n}\right)$.

Proof. For $y>0$ and $N \in \mathbb{N} \cup\{0\}$ we have

$$
\begin{aligned}
\int_{\sigma-i \infty}^{\sigma+i \infty} & M_{q}^{N}(f)(s) y^{-s} d s \\
& =-\frac{1-q}{\log q} \sum_{|n| \leq N} \int_{\sigma-i \infty}^{\sigma+i \infty} M(f)\left(s+\frac{2 \pi i n}{\log q}\right) y^{-s} d s \\
& =-\frac{1-q}{\log q} \sum_{|n| \leq N} y^{i \frac{2 \pi n}{\log q}} \int_{\sigma-i \infty}^{\sigma+i \infty} M(f)(s) y^{-s} d s \\
& = \begin{cases}-\frac{2 \pi i(1-q)}{\log q} f(y) \cdot(2 N+1) & \text { if } y=q^{n}(n \in \mathbb{Z}), \\
-\frac{2 \pi i(1-q)}{\log q} f(y) \cdot \frac{\sin \left((2 N+1) \pi \frac{\log y}{\log q}\right)}{\sin \left(\pi \frac{\log y}{\log q}\right)} & \text { otherwise. }\end{cases}
\end{aligned}
$$

Hence (A.1) follows. Next assume that $M_{q}^{N}(f)(\sigma+i t)$ converges absolutely and uniformly for $t$ in any compact subset of $\mathbb{R}$ as $N \rightarrow \infty$. The Fourier transform of the function

$$
\varphi(x):=M(f)\left(\sigma+\frac{2 \pi i}{\log q} x\right) \quad(x \in \mathbb{R})
$$

is given by

$$
\begin{aligned}
\widehat{\varphi}(\xi) & :=\int_{-\infty}^{\infty} M(f)\left(\sigma+\frac{2 \pi i}{\log q} x\right) e^{-2 \pi i x \xi} d x \\
& =-\frac{q^{\xi \sigma} \log q}{2 \pi i} \int_{\sigma-i \infty}^{\sigma+i \infty} M(f)(s) q^{-\xi s} d s=-f\left(q^{\xi}\right) q^{\xi \sigma} \log q \quad(\xi \in \mathbb{R}) .
\end{aligned}
$$

Hence, by the Poisson summation formula $\sum_{n \in \mathbb{Z}} \varphi(x+n)=\sum_{n \in \mathbb{Z}} \widehat{\varphi}(n) e^{2 \pi i n x}$ $(x \in \mathbb{R})$, we get

$$
\begin{aligned}
\lim _{N \rightarrow \infty} M_{q}^{N}(f)(\sigma+i t) & =-\frac{1-q}{\log q} \sum_{n \in \mathbb{Z}} \varphi\left(\frac{t \log q}{2 \pi}+n\right) \\
& =-\frac{1-q}{\log q} \sum_{n \in \mathbb{Z}} \widehat{\varphi}(n) e^{2 \pi i n \cdot \frac{t \log q}{2 \pi}} \\
& =(1-q) \sum_{n \in \mathbb{Z}} q^{n(\sigma+i t)} f\left(q^{n}\right)=M_{q}(f)(\sigma+i t) \quad(t \in \mathbb{R}) .
\end{aligned}
$$


Remark A.2. When $N=0$, (A.1) gives just the classical Mellin inversion formula. However, when $N \geq 1$, (A.1) does not give the expression of $f(y)$ for $y=q^{n /(2 N+1)}$ with $n /(2 N+1) \notin \mathbb{Z}$.

REMARK A.3. If $f$ is written as the series $f(y)=\sum_{n>1} a_{n} e^{-2 \pi n y}(y>0)$ with $\left|a_{n}\right|=O\left(n^{\alpha}\right)$ as $n \rightarrow \infty$ for some $\alpha>0$, then $f(y)=O\left(y^{-\alpha-1}\right)$ as $y \rightarrow 0, f(y)=O\left(e^{-2 \pi y}\right)$ as $y \rightarrow \infty$ and $M(f)(s)=(2 \pi)^{-s} \Gamma(s) L(s)$ for $\operatorname{Re}(s)>\alpha+1$ with $L(s):=\sum_{n \geq 1} a_{n} n^{-s}(\operatorname{Re}(s)>\alpha+1)$. Then, for a fixed $\sigma>\alpha+1$, one sees that $M_{q}^{N}(f)(\sigma+i t)$ converges absolutely and uniformly for $t$ in any compact subset of $\mathbb{R}$ as $N \rightarrow \infty$. Actually, by Stirling's formula and the boundedness of $(2 \pi)^{-s} L(s)$ on $\operatorname{Re}(s)=\sigma$, for $t \in[-R, R](R>0)$ and $|n|>R|\log q| / 2 \pi$ we can estimate

$$
\left|M(f)\left(\sigma+i t+\frac{2 \pi i n}{\log q}\right)\right| \leq C_{\sigma}\left|t+\frac{2 \pi n}{\log q}\right|^{\sigma-1 / 2} e^{-\frac{\pi}{2}\left|t+\frac{2 \pi n}{\log q}\right|} \leq C_{\sigma}^{\prime} e^{-\left(\frac{2 \pi|n|}{|\log q|}-R\right)}
$$

with some $C_{\sigma}, C_{\sigma}^{\prime}>0$. Hence, in this case,

$$
\lim _{N \rightarrow \infty} M_{q}^{N}(f)(\sigma+i t)=M_{q}(f)(\sigma+i t) \quad(t \in \mathbb{R}) .
$$

B. Appendix- $\zeta^{(q)}(s)$ gives a true $q$-analogue of $\zeta(s)$. In this appendix, we prove that $\zeta^{(q)}(s)$ gives a true $q$-analogue like the $q$-analogue $\zeta_{q}(s)$ of $\zeta(s)$ introduced in [7] (see also [9]). Namely, we show the following:

Proposition B.1. For all $s \in \mathbb{C}$, we have

$$
\lim _{q \rightarrow 1} \zeta^{(q)}(s)=\pi^{-s / 2} \Gamma\left(\frac{s}{2}\right) \zeta(s) .
$$

Proof. Recall the original property of the $q$-Mellin transform. If $f$ is a moderate asymptotic function of order $\left(i_{0}, j_{0}\right)$, then for $-i_{0}<\operatorname{Re}(s)<-j_{0}$ we have $M_{q}(f)(s) \rightarrow M(f)(s)$ as $q \rightarrow 1$, i.e.,

$$
\lim _{q \rightarrow 1} \sum_{n \in \mathbb{Z}} q^{n(s-1)} f\left(q^{n}\right)\left(q^{n}-q^{n+1}\right)=\int_{0}^{\infty} y^{s-1} f(y) d y .
$$

More precisely,

$$
\lim _{q \rightarrow 1} \sum_{n \geq 1} q^{n(s-1)} f\left(q^{n}\right)\left(q^{n}-q^{n+1}\right)=\int_{0}^{1} y^{s-1} f(y) d y \quad\left(\operatorname{Re}(s)>-i_{0}\right)
$$

and

$$
\lim _{q \rightarrow 1} \sum_{n \leq-1} q^{n(s-1)} f\left(q^{n}\right)\left(q^{n}-q^{n+1}\right)=\int_{1}^{\infty} y^{s-1} f(y) d y \quad\left(\operatorname{Re}(s)<-j_{0}\right) .
$$


Using these formulas, we rewrite the expression of $\zeta^{(q)}(s)$. We have

$$
\begin{aligned}
\zeta^{(q)}(s)= & \sum_{n \in \mathbb{Z}} q^{n(s-1)}\left(\theta\left(q^{2 n}\right)-1\right)\left(q^{n}-q^{n+1}\right) \\
= & (1-q)(\theta(1)-1)+\sum_{n \leq-1}\left(q^{-n s}+q^{n(s-1)}\right)\left(\theta\left(q^{2 n}\right)-1\right)\left(q^{n}-q^{n+1}\right) \\
& +(1-q)\left(\frac{q^{s-1}}{1-q^{s-1}}-\frac{q^{s}}{1-q^{s}}\right)
\end{aligned}
$$

for $\operatorname{Re}(s)>1$. Since $\theta\left(y^{2}\right)-1$ is a moderate asymptotic function of order $(-1, *)$, the above equation holds for all $s \in \mathbb{C}$ and

$$
\lim _{q \rightarrow 1} \zeta^{(q)}(s)=\int_{1}^{\infty}\left(y^{-s}+y^{s-1}\right)\left(\theta\left(y^{2}\right)-1\right) d y+\frac{1}{s(s-1)} \quad(s \in \mathbb{C}) .
$$

Also,

$$
\begin{aligned}
\pi^{-s / 2} \Gamma\left(\frac{s}{2}\right) \zeta(s) & =\int_{0}^{\infty} y^{s-1}\left(\theta\left(y^{2}\right)-1\right) d y \\
& =\int_{1}^{\infty}\left(y^{-s}+y^{s-1}\right)\left(\theta\left(y^{2}\right)-1\right) d y+\frac{1}{s(s-1)}
\end{aligned}
$$

for $\operatorname{Re}(s)>1$. Hence the assertion is obtained by analytic continuation.

REMARK B.2. The following table gives present results on $q$-analogues $\zeta_{q}(s)$ and $\zeta^{(q)}(s)$ of $\zeta(s)$. The "+" in the last column means that several special values of $\zeta_{q}(s)$ can be evaluated explicitly by $q$-Bernoulli numbers (see [7], [9]), and the question mark in that column indicates that no special values of $\zeta^{(q)}(s)$ have been calculated. This is a problem for future research.

Table 1. Present results

\begin{tabular}{rccc}
\hline & True $q$-analogue & Functional equation & Special values \\
\hline$\zeta_{q}(s):$ & + & $?$ & + \\
$\zeta^{(q)}(s):$ & + & + & $?$ \\
\hline
\end{tabular}

REMARK B.3. In view of the transformation rule given by (2.6), as in the proof of Proposition B.1, we can also see that the $q$-series $\Lambda_{q}(s, F)$ defined by $(2.3)$ is a true $q$-analogue of the completed L-function $\Lambda(s, F)$ of Remark 2.2 .

\section{References}

[1] D. Bump, Automorphic Forms and Representations, Cambridge Univ. Press, Cambridge, 1997. 
[2] A. Fitouhi, N. Bettaibi and K. Brahim, The Mellin transform in quantum calculus, Constr. Approx. 23 (2006), 305-323.

[3] S. Haran, The Mysteries of The Real Prime, London Math. Soc. Monogr. 25, Clarendon Press, Oxford, 2001.

[4] S. Haran, N. Kurokawa and M. Wakayama, Jackson-Mellin's transform of modular forms and q-zeta functions, Kyushu J. Math., to appear.

[5] E. Hecke, Über die Bestimmung Dirichletscher Reihen durch ihre Funktionalgleichung, Math. Ann. 112 (1936), 664-699.

[6] H. Iwaniec, Topics in Classical Automorphic Forms, Amer. Math. Soc., 1997.

[7] K. Kaneko, N. Kurokawa and M. Wakayama, A variation of Euler's approach to values of the Riemann zeta function, Kyushu J. Math. 57 (2003), 175-192.

[8] E. C. Titchmarsh, The Theory of the Riemann Zeta-Function, 2nd ed., rev. by D. R. Heath-Brown, Oxford Univ. Press, Oxford, 1986.

[9] M. Wakayama and Y. Yamasaki, Integral representations of q-analogues of the Hurwitz zeta function, Monatsh. Math. 149 (2006), 141-154.

[10] A. Weil, Über die Bestimmung Dirichletscher Reihen durch Funktionalgleichungen, Math. Ann. 168 (1967), 149-156.

Graduate School of Mathematics

Kyushu University

Hakozaki, Fukuoka 812-8581, Japan

E-mail: m-mera@math.kyushu-u.ac.jp 